\title{
Hysteroscopic septum resection in patients with recurrent abortions and infertility
}

\author{
Davut Güven, Kadir Bakay*, Serkan Kuruoğlu, Ayşe Özdemir \\ Department of Obstetrics and Gynecology, 19 Mayis University School of Medicine, Samsun, Turkey \\ Email: drkadirbakay@gmail.com, dguven@omu.edu.tr, drserkan08@gmail.com, aysezehra.ozdemir@hotmail.com
}

Received 15 May 2012; revised 20 June 2012; accepted 6 July 2012

\begin{abstract}
Objective: The aim of this study was to assess the achievement of pregnancy as well as the reproductive outcome after hysteroscopic septum resection in women with recurrent abortions or infertility. Design: retrospective, clinical trial. Setting: University hospitals. Patient(s): Fourty women with a diagnosis of uterine septum who had a history of pregnancy loss or infertility. They were randomized into two groups. Group I includes 18 infertile women and Group II includes 22 recurrent miscarriage women. Intervention(s): Hysteroscopic metroplasty was performed on all patients in both groups. Result(s): 40 patients who were diagnosed with a partial septate uteri had hysteroscopic septum resection and were divided into two groups: Group I (18 patients) presented with infertility and Group II (22 patients) with recurrent abortions. The mean post-operative follow up period was 24 months. Group I, 12 patients $(66.6 \%)$ and Group II, 17 patients (77.2\%) achieved pregnancy, with respective abortion rates of $2(11.1 \%)$ and 4 (18.1\%). Conclusion(s): Using hysteroscopy to perform metroplasty on septate uteri makes the procedure safer, easier, and less complicated. This procedure is recommended for all cases diagnosed with an uterine septum.
\end{abstract}

Keywords: Hysteroscopy; Infertility; Recurrent Abortion; Septum Resection

\section{INTRODUCTION}

Uterine Septum as an anomaly may be associated with poor reproductive performance, including higher incidence of abortion, premature delivery, and infertility. Although the septum is usually restricted to the uterine corpus, it may extend through the cervix and vagina. In such cases visual inspection and pelvic examination shows a complete longitudinal vaginal septum and cer-

"Corresponding author. vical duplication that is usually misdiagnosed as uterus didelphys [1].

The main and absolute indication for the treatment of patients with septate uteri is a reproductive history of recurrent abortions or fetal loss. However, a group of patients with septate uteri may present with infertility, primary or secondary, and this will be discovered only during the infertility work-up. Minimally invasive procedures have many benefits compared with traditional procedures, including lower costs, shorter hospital stays, and shorter recovery time. The development of operative hysteroscopy has simplified the treatment of these malformations, which were previously treated by laparotomy. Surgical hysteroscopy is no longer performed with rigid scissors attached to a channel. Today, these metroplasties use monopolar instruments.

Bipolar instruments, which have been introduced more recently, seem to be as effective and result in less morbidity [2]. Hysteroscopic metroplasty in this group is applied mainly as a prophylactic procedure to prevent spontaneous abortions and complications during labour [3].

\section{MATERIAL AND METHODS}

Fourty subfertile patients who underwent hysteroscopic septum resection at the center for Department Obstetrics and Gynecology of 19 Mayis University School of Medicine between January 2005 and March 2009 were included in the study. The diagnosis of uterin septum was accomplished by performing transvaginal ultrasonography after hysterosalpingography in all patients.

The hysteroscopic operation of the uterine septum was scheduled in the early proliferative phase. The operation was performed under general anaesthesia and laparoscopic control was not performed during operation.

Inclusion criteria were determined locally and consisted of all patients with a complete or partial septate uterus who had a history of at least one first-trimester or second-trimester pregnancy loss and who had undergone preoperative evaluation to exclude other causes of re- 
productive failure. Two or more miscarriages in the last 2 years, premature delivery, no endocrine or other disorders as in the previous group.

The cervix was dilated up to $9 \mathrm{~mm}$ and the uterine cavity was distended with a non-conductive solution. Fluid balance was recorded in all patients. When a partial uterin septum was present, resection was initiated at its lower margin, after the tubal ostia had been visualized. The resection continued upwards and was considered complete when the area between the tubal ostia was converted to a line. The hysteroscopic septum resection was performed under general anaesthesia. Postoperatively, a second-look hysteroscopy and hysterosalpingography was not deemed necessary instead patients were reevaluated by transvaginal ultrasonography 2 months after the operation.

\section{RESULTS}

The main reproductive history data are shown (before septum resection) in Table 1. In the infertile Group I, 12 (66.6\%) achieved pregnancy. 2 of the 12 pregnancies (11.1\%) ended in miscarriage; 1 (5.5\%) ended as preterm delivery; 9 of the 18 pregnancies (50\%) ended as term deliveries, 6 (33.3\%) of the infertile patients were unable to become pregnant. In the recurrent abortion Group II, 17 patients (77.2\%) achieved pregnancy. 4 (18.1\%) ended in miscarriage; 2 pregnancies (9\%) ended as pre-term delivery; $11(64.7 \%)$ pregnancies ended as term deliveries. 5 (22.7\%) out of 22 patients did not achieve pregnancy after hysteroscopic septum resection (Table 2).

There were no operative complications such as uterine perforation, hyponatremia, hemorrhage, bowel or bladder injur in the study group.

\section{DISCUSSION}

Minimally invasive procedures have many benefits when

Table 1. Reproductive history before septum resection.

\begin{tabular}{ccc}
\hline & Group I (n: 18) & Group II (n: 22) \\
\hline Patients (n) & 18 & 22 \\
Age in year (mean \pm SD) & $27 \pm 3$ & $29 \pm 5$ \\
$\begin{array}{c}\text { Duration in infertility in a } \\
\text { year (mean } \pm \text { SD) }\end{array}$ & $5.2 \pm 3.1$ & 0 \\
Pregnancies (n) & 0 & 56 \\
Abortions (n) & 0 & $56(100 \%)$ \\
Intra uterine fetal death (n) & 0 & 2 \\
Pre-term deliveries (n) & 0 & 0 \\
Term deliveries (n) & 0 & 0 \\
\hline
\end{tabular}

Table 2. Reproductive history after septum resection (the mean period of follow up was 24 months).

\begin{tabular}{cccc}
\hline & Group I & Group II & Total \\
\hline Patients (n) & 18 & 22 & 40 \\
Pregnant (n [\%]) & $12(66.6 \%)$ & $17(77.2 \%)$ & $29(72.5 \%)$ \\
Abortions (n [\%]) & $2(11.1 \%)$ & $4(18.1 \%)$ & $6(15.0 \%)$ \\
Intra uterine fetal death (n [\%]) & 0 & 0 & 0 \\
Pre-term deliveries (n [\%]) & $1(5.5 \%)$ & $2(9.0 \%)$ & $3(7.5 \%)$ \\
Healthy pregnancy (n [\%]) & $9(50 \%)$ & $11(64.7 \%)$ & $20(50 \%)$ \\
Non pregnant women & $6(33.3 \%)$ & $5(22.7 \%)$ & $11(28 \%)$ \\
\hline
\end{tabular}

compared with traditional procedures, including shorter hospital stays, lower cost, and shorter recovery time. Daly et al. (1989) reported that 7 (53.8\%) out of 13 patients with infertility conceived after septum resection and, more recently, Goldenberg et al. (1995) have observed pregnancies after hysteroscopic metroplasty in 18 (54\%) out of 34 patients with uterine septum and infertility.

Thus, the chance of conception in patients with septate uteri and infertility seems to be similar to those of the general infertile population either with or without septum resection. This may also be an indirect sign that uterine septum is not an infertility factor in itself. However, the treatment has a beneficial effect on pregnancy outcomes [3]. Although congenital uterine malformations are associated with poor reproductive performance, each type may have a different impact on reproduction [4]. Unicornuate uterus and uterus didelphys seem to have a similar effect on pregnancy outcome, since uterus didelphys may be considered as a symmetrical duplication of a unicornuate uterus [4-6].

The performance of hysteroscopic metroplasty in patients with primary infertility is still a subject of debate, with some groups recommending treatment and others not. Generally treatment is considered in women in whom assisted conception is being contemplated.

Considering that the procedure used in this study is simple and without complications, this is probably justified, as the obstetric outcome in pregnant patients with a uterin septum is worse [7].

S. Venturoli observed that, 141 patients who had a partial uterine septum and had undergone hysteroscopic metroplasty (class Vb, American Society for Reproductive Medicine, ASRM) were divided into two groups: Group I (69 patients) presented with infertility and Group II (72 patients) with recurrent abortions were observed that after operation, 36 of Group I patients (52.1\%) and 38 of Group II patients (52.7\%) achieved pregnancy, with respective abortion rates of $20 \%$ and $25 \%$ [8]. 


\section{CONCLUSION}

Hysteroscopic septum resection is a minimally invasive procedure and it appears to be related with a significant improvement in achieving pregnancy in women with a history of recurrent abortions. Traditional metroplasty is well known to cause pelvic adhesions thus further complicating the case.

\section{REFERENCES}

[1] Parsanezhad, M.E., Alborzi, S., Zarei, A., Dehbashi, S., Shirazi, L.G., Rajaeefard, A. and Schmidt, E.H. (2006) Hysteroscopic metroplasty of the complete uterine septum, duplicate cervix, and vaginal septum. Fertility \& Sterility, 85, 5.

[2] Fernandez, H., Gervaise, A. and de Tayrac, R. (2000) Operative hysteroscopy for infertility using normal saline solution and a coaxial bipolar electrode: A pilot study. Human Reproduction, 15, 1773-1775. doi:10.1093/humrep/15.6.1425

[3] Grimbizis, G., Camus, M., Clasen, K., Tournaye, H., De Munck, L. and Devroey, P. (1998) Hysteroscopic septum resection in patients with recurrent abortions or infertility.
Human Reproduction, 13, 1188-1193.

[4] Buttram, C.V. (1983) Müllerian anomalies and their management. Fertility \& Sterility, 40, 159-163.

[5] Moutos, M.D., Damewood, D.M., Schlaff, D.W. and Rock, A.J. (1992) A comparison of the reproductive outcome between women with a unicornuate uterus and women with a didelphic uterus. Fertility \& Sterility, $\mathbf{5 8}$ 88-93.

[6] Marcus, S., Al-Shawaf, T. and Brinsden, P. (1996) The obstetric outcome of in vitro fertilization and embryo transfer in women with congenital uterine malformation. American Journal of Obstetrics \& Gynecology, 175, 8589. doi:10.1016/S0002-9378(96)70255-7

[7] Zikopoulos, K.A., Kolibianakis, E.M., Tournaye, H., de Munck, L., Devroey, P. And Camus M. (2003) Hysteroscopic septum resection using the Versapoint system in subfertile women. Reproductive BioMedicine Online, 7, 365-367.

[8] Venturoli, S., Colombo, F.M., Vianello, F., Seracchioli, R., Possat, G. and Paradisi R. (2002) A study of hysteroscopic metroplasty in 141 women with a septate uterus. Archives of Gynecology and Obstetrics, 266, 157-159. doi:10.1007/s004040100217 